\title{
Instruments and Methods
}

\section{Precise dielectric profiling of ice cores: a new device with improved guarding and its theory}

\author{
Frank Wilhelms, ${ }^{1}$ Josef Kipfstuhl, ${ }^{1}$ Heinz Miller, ${ }^{1}$ Klaus Heinloth, ${ }^{2}$ John Firestone ${ }^{1}$ \\ ${ }^{1}$ Alfred-Wegener-Institut für Polar-und Meeresforschung, Columbusstrasse, D-27568 Bremerhaven, Germany \\ ${ }^{2}$ Physikalisches Institut der Universität Bonn, Nussallee 12, D-53115 Bonn, Germany
}

\begin{abstract}
Dielectric profiling (DEP), a capacitive method for measuring an ice core's electrical conductivity and permittivity, has been in use for several years. We have built an improved DEP instrument with a modified guarded-electrode geometry whose capacitance can be determined from potential theory. The new instrument can measure an ice core's conductivity and permittivity to within a few per cent error, a high enough accuracy to infer its density using well-known models.
\end{abstract}

\section{INTRODUGTION}

Electrical methods can quickly provide estimates of the impurity content of an ice core with a resolution of a few millimeters. The electrical conductivity method (ECM; Hammer, 1977; Hammer and others, 1980) can detect volcanic events and measure acidity. Dielectric profiling (DEP) as a non-destructive method additionally yields estimates of salt and ammonia concentrations (Moore and others, 1992, 1994). We used the DEP method in the field to find well-known volcanic horizons and to estimate the age of ice being taken on a traverse in north Greenland in 1995.

ECM measures an ice core's d.c. conductivity (Hammer, 1980). The ice core is first microtomed, then two electrodes with a potential difference of $1000-2000 \mathrm{~V}$ are dragged across its freshly cut surface. The resulting microampere current largely reflects the hydrogen ion concentration $\left[\mathrm{H}^{+}\right]$. We did not use this method during our traverse as we did not wish to cut up our ice cores.

The DEP method measures an ice core's a.c. capacitance and conductance (Moore and Paren, 1987) at 42 frequencies between $20 \mathrm{~Hz}$ and $300 \mathrm{kHz}$. The core is placed between two curved electrodes which form a capacitor with the ice core as its dielectric. In our new device the electrodes are driven by a $250 \mathrm{kHz}$ signal, and their a.c. capacitance and conductance are measured with an auto-balancing bridge. At this frequency the ice core's conductivity and permittivity can be calculated immediately once the free air capacitance between the electrodes is known.

While trying to replicate the DEP instruments of Moore and Paren (1987) and Moore (1993), we were unable to obtain an exact solution of the instrument's electrical properties, either its potential function or its free air capacitance, because its electrodes do not encircle the ice core (Wilhelms, 1996). Moore and Maeno (1991) could only give a rough estimate of the free air capacitance of their DEP electrode geometry. By altering the electrode geometry, however, we obtain an exact solution using Green's functions, and by modifying the guard electrodes we eliminate stray capacitances. Thus we are able to quantify precisely and improve the device's performance. Our DEP instrument is the consequence of the guarded capacitor method carefully applied to the ice core's curved geometry.

\section{THE GUARDED GAPACITOR METHOD AND DEP}

A DEP instrument is essentially a calibrated scanning capacitor that measures a section of ice core by having it serve as its dielectric. To measure just the bulk dielectric properties of an ice core, the design of a DEP instrument must circumvent three main sources of error.

Polar ice cores are normally measured at temperatures above $-30^{\circ} \mathrm{C}$, but should not be stored at temperatures above $-10^{\circ} \mathrm{C}$ (Reynolds, 1985). Above $-25^{\circ} \mathrm{C}$, an ice core's surface conductivity becomes significant (Petrenko, 1993). Camp and others (1969), for example, measured a surface conductivity that was $20 \%$ of the bulk conductivity in a polycrystalline ice sample at $-11.6^{\circ} \mathrm{C}$. A DEP instrument avoids surface currents using guard electrodes which force the electric field vector to enter and exit the sample perpendicular to its surface.

In a parallel-plate capacitor, guard electrodes are usually sufficient to avoid external stray capacitance. The curved electrodes of a DEP instrument, however, approach each other at the edges, so that a significant surface charge is influenced from the outside to some distance beyond the edges. A DEP instrument eliminates this exterior stray capacitance by external, electromagnetic shielding of the LO electrode (cf. Fig. 1).

When an ice sample contacts a metal electrode, the ice and metal partially exchange charges (Petrenko, 1993). This exchange can be eliminated by inserting an insulating blocking layer between the sample and the electrodes (Mounier and Sixou, 1969; Gross and others, 1975), for example a thin film of Teflon (Gross and others, 1980). Alternatively, in a modern DEP instrument, the blocking layer is achieved by anodizing the aluminum electrodes (Wolff and others, 1995).

Figure 1 shows a guarded, electrically shielded capacitor 


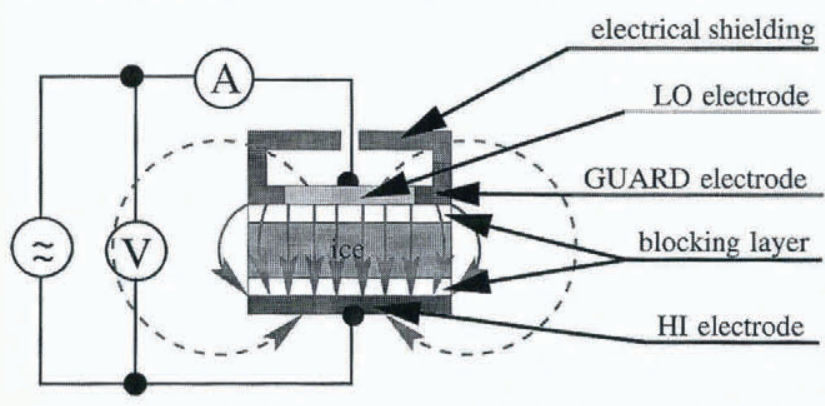

Fig. 1. Measurement of a guarded capacitor with blocking layers. The LO and HI electrodes connect to the corresponding inputs of a Hewlett Packard 4284A Precision LCR Meter. The electrical shielding and the guard electrode connect to a physical ground, while the LO electrode connects to a virtual ground at ground potential.

with blocking layers. Figures 2 and 3 show its realization as a DEP instrument with curved electrodes.

The first DEP instrument (Moore and Paren, 1987) had fixed lower and upper electrodes, a $108^{\circ} \mathrm{HI}$ electrode in which the ice core was placed and a $108^{\circ} \mathrm{LO}$ electrode, broken into $5 \mathrm{~cm}$ segments and scanned electrically using relays. A later version uses $2 \mathrm{~cm}$ segments (Wolff and others, 1995). Curved guard plates were fitted at both ends, and flat guard plates were mounted alongside the HI and LO electrodes.

A second high-resolution $50 \mathrm{kHz}$ DEP instrument had a fixed $108^{\circ} \mathrm{HI}$ electrode and a single, straight, guarded, $3 \mathrm{~mm}$ wide LO electrode which was moved by hand over the ice core (Moore, 1993). A later version, which inspired the present instrument, uses a curved LO electrode (Friedmann and others, 1995). Both instruments give a qualitative measure of changes in an ice core's high-frequency conductivity. Because of their geometry, the electrical properties,

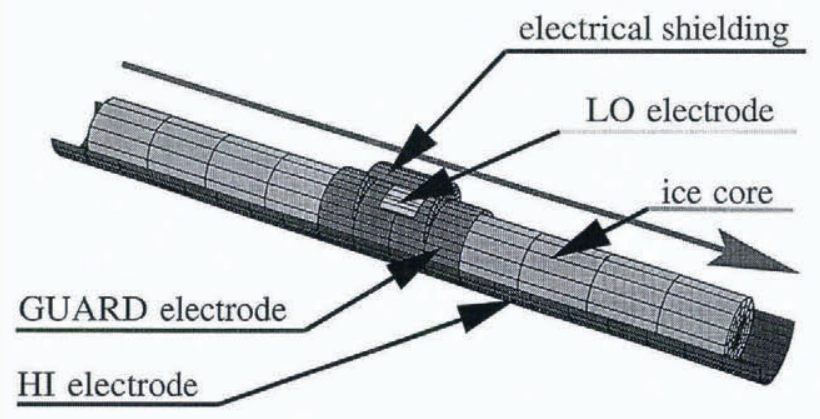

Fig. 2. Schematic of the DEP instrument.

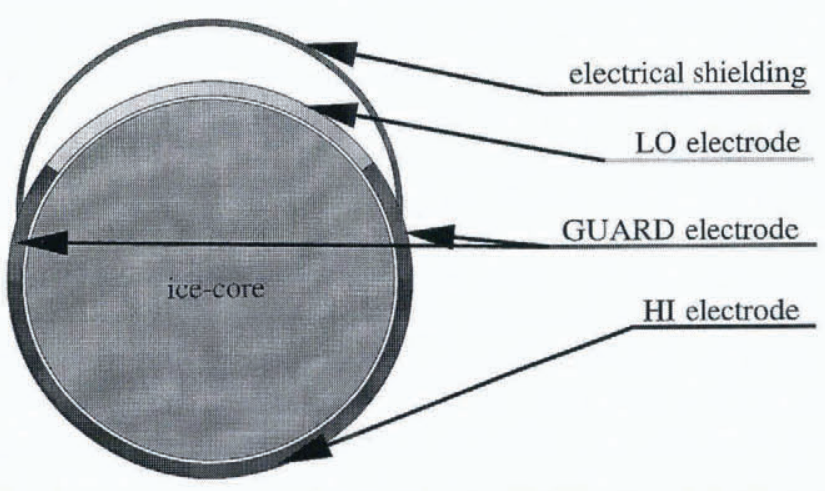

Fig. 3. Schematic of the electrode configuration. such as the potential function or the free air capacitance, of the first four DEP instruments are inexactly known, even though the free air capacitance can be estimated (Moore and Maeno, 1991).

\section{THE NEW DEP INSTRUMENT: THEORY AND DESIGN}

To know its electrical properties more precisely, we designed and tested a high-resolution instrument with an improved set of electrodes. Its potential distribution is easily calculated using Green's functions, which allows substantially more accurate dielectric measurements of an ice core.

\section{Instrument design}

The instrument has a single, $108^{\circ}, 1 \mathrm{~cm}$ wide $\mathrm{LO}$ electrode, placed in the middle of a $70 \mathrm{~cm}$ long guard electrode of anodized aluminum half-tube and shielded on the outside. The $\mathrm{HI}$ electrode in which the ice core rests is a $350 \mathrm{~cm}$ long halftube. The $\mathrm{HI}$ electrode is fixed to an aluminum frame and the guarded LO electrode glides over the ice on rails (cf. Fig. 4).

A stepping motor drives the LO electrode the length of the ice core. As the electrode scans the surface in $2-5 \mathrm{~mm}$ steps, a direct connection feeds its signal to the balancing bridge. The single electrode and simple connection avoid two complications inherent in the very first DEP instruments: the resolution of the instrument is not limited by a fixed number of relays, and its capacitance is not disturbed by the relays or their wiring. These stray capacitances are large and unknown, and may exceed the $100 \mathrm{fF}$ free air capacitance between the LO and HI electrodes; they may also disturb the symmetry of the balancing bridge.

Stray capacitances are a serious problem as they add to the free air capacitance attributed to the electrodes. This decreases the fraction of the total measured capacitance influenced by the ice-core sample and thus underestimates its dielectric properties. It is extremely difficult to deduct these stray capacitances through additional measurements without extensive decomposition of the instrument's capacitances, for example by repeated measurement of samples with known dielectric values. It is much easier and more assuring to avoid significant stray capacitances in the first place, which is what our new instrument has done, in theory, by design and in practice.

\section{Calculation of the potential distribution}

Because the instrument's guard electrode is very long compared to the LO electrode, the potential distribution can be calculated in plane polar coordinates. Because the wavelength of the excitation signal (no more than $1 \mathrm{MHz}$ ) is three orders of magnitude longer than the $100 \mathrm{~mm}$ diameter of the instrument, the distribution can be treated as electrostatic. It can then be accurately solved using Green's functions and the method of mirror charges, assuming the potential is known on the entire surface of the ice core (Wilhelms, 1996). Figure 5 shows the calculated potential distribution.

\section{Proof of guarding}

From the potential, Wilhelms (1996) calculated the components of the electrical field normal and tangential to the ice core's surface. The ratio of the tangential to the normal component over the area scanned by the LO electrode is less 


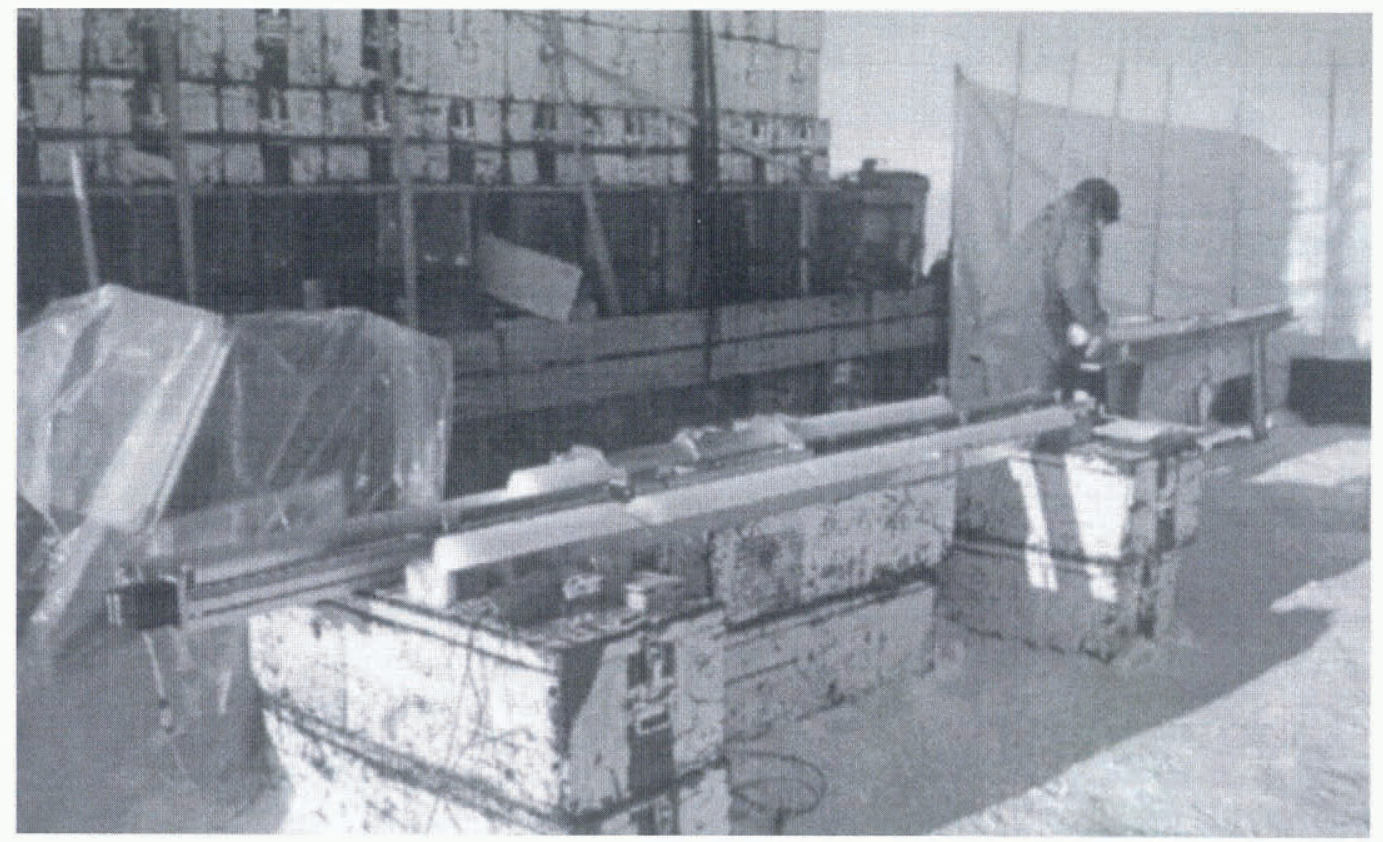

Fig. 4. The portable DEP instrument in operation during the North Greenland Traverse, 1995.
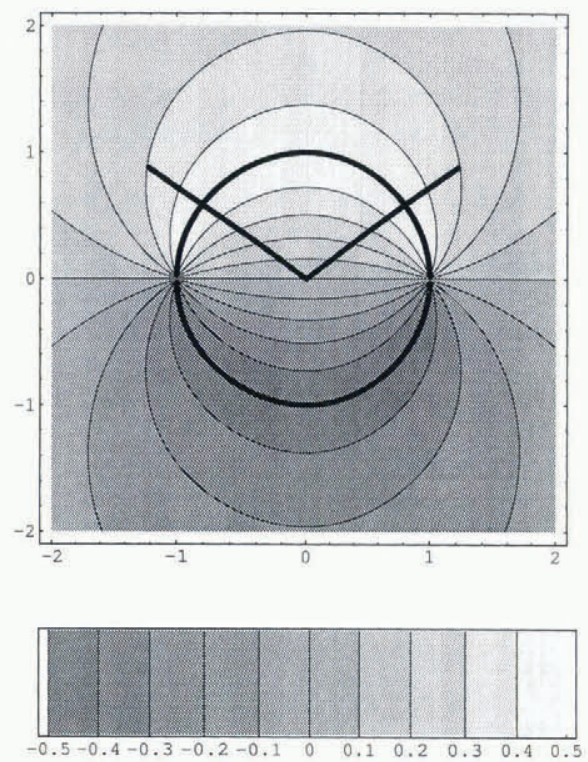

Fig. 5. Calculated potential distribution both with and without an ice-core sample. The electrodes and the upper (LO) electrode angle of $108^{\circ}$ are drawn with a solid line. The upper ( LO) and lower ( HI) electrodes are at 1/2 unit positive and 1/2 unit negative potentials. The equipotential lines are in 1/10 unit potential steps from the horizontal zero line. The axes are in units of ice-core radians.

than 0.03 if the diameter of the ice core is at least 0.98 electrode radians, our estimate of its minimum size. Assuming the ratio of the tangential and radial conductances is approximately 0.2 (Camp and others, 1969), the ratio of the corresponding currents will be less than 0.01 . The guarding will thus reduce the surface currents to less than $1 \%$, which is satisfactory.

\section{Free air capacitance}

Once the potential distribution is known, the electrical properties can be determined. The radial derivative of the potential function times the permittivity gives the surface charge density. Its integral over the LO electrode area gives the total charge. The total charge divided by the potential differences yields the capacitance. The free air capacitance, $C_{0}$, between the $\mathrm{LO}$ and $\mathrm{HI}$ electrodes is: $C_{0}=$ $\left(2 \epsilon_{0} l / \pi\right) \ln |(\cot \xi) / 2|$, where $\epsilon_{0}$ is the permittivity constant, $l$ is the width of the LO electrode, and $\xi$ is the angle in Figure 5 subtended by a guard electrode from the horizontal to the LO electrode (Wilhelms, 1996). With $l=1 \mathrm{~cm}$ and $\xi=\pi / 5, C_{0}=63.4 \mathrm{fF}$, which agrees very well with the instrument's measured capacitance of $63.7 \pm 1.3 \mathrm{fF}$. This suggests the assumption of plane polar geometry is reasonable and that stray capacitances are insignificant. Failure of either assumption would increase the measured free air capacitance, the former because of end effects.

\section{RESULTS AND DISCUSSION}

The improved DEP instrument obtains dielectric properties (Fig. 6) which agree very well with those calculated from the conductance value of $\sigma_{\infty}=1.9 \times 10^{-5} \mathrm{~S} \mathrm{~m}^{-1}$ for $-20^{\circ} \mathrm{C}$, the conductance value for Greenlandic glacial ice measured by Paren (1973, fig. 1), with a parallel plate system.

The redesigned guard electrodes allow the measurement of precise conductivity values even at relatively high sample temperatures (here $-10^{\circ} \mathrm{C}$ ), temperatures at which surface currents would otherwise become excessive. Assuming the Looyenga (1965) mixing model and a permittivity for pure ice of 3.17, the instrument yields ice-core densities that agree with precise densities measured by $\gamma$ absorption (Wilhelms, 1996; Fig. 7).

Despite its relatively broad width, the LO electrode's improved geometry allows the instrument to reproduce many fine variations in the density (Fig. 8). Until now, such details could only be resolved by continuous $\gamma$-absorption measurements.

A calibration of the instrument's HP 4284A LCR Meter suggests the high-frequency permittivity determined for an ice core has an error of $4.5 \%$. The calibration suggests the conductivities have an error of $8-15 \%$ for core sections from below and above $30 \mathrm{~m}$ depth, respectively (Wilhelms, 1996). 

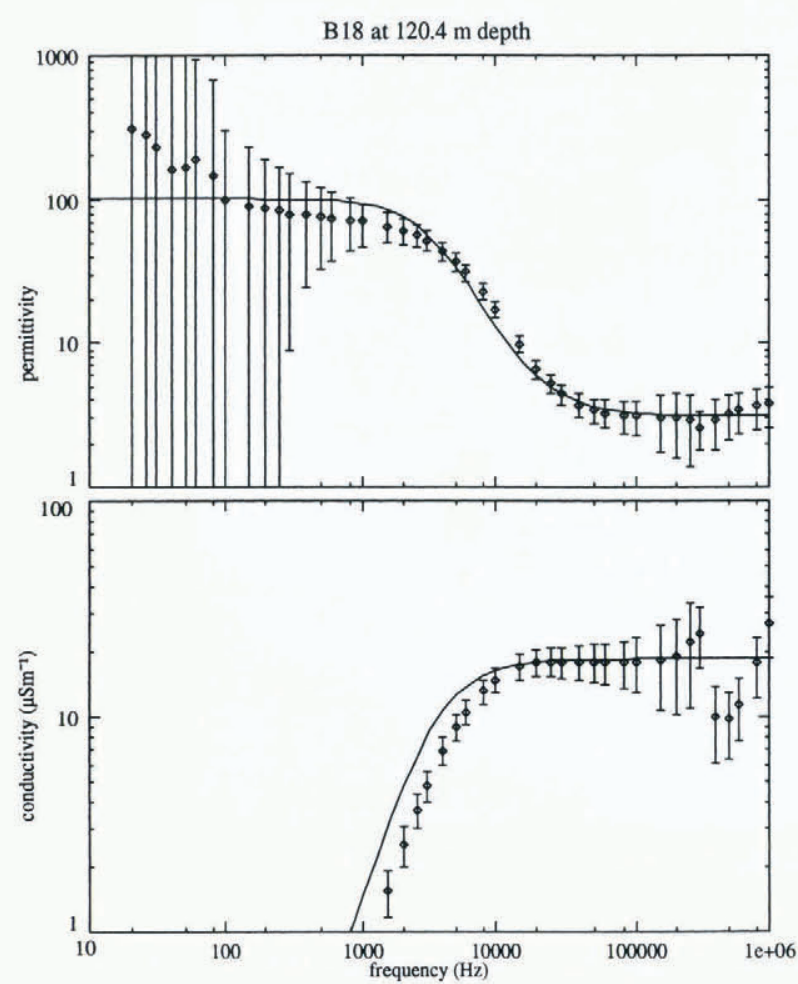

Fig. 6. Measurements with error bars of ice from a north Greenland ice core compared to the smooth electrical properties of pure glacier ice measured by Paren (1973) with a parallel plated capacitor set-up assuming the model of Faccard (1959) and compensating for a density effect predicted by the Looyenga (1965) mixing model.

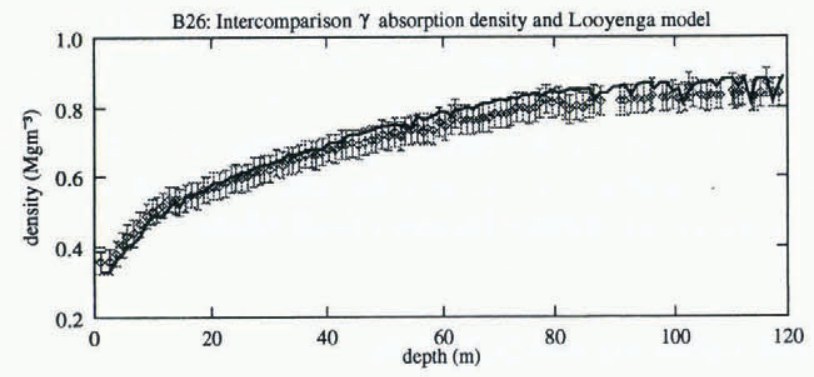

Fig. 7. Density predicted from permittivity by the Looyenga mixing model (shown with roughly $15 \%$ error bars) compared to density measured by $\gamma$ absorption with an error of $0.006 \mathrm{Mg} \mathrm{m}^{-3}$ (solid line).

A better bridge would increase the accuracy of the measurements, as would a longer LO electrode. A longer LO electrode, however, would decrease the resolution.

The much higher precision of our DEP instrument opens up new possibilities for analyzing the initial firn section of ice cores. We plan to extend the method to low frequencies through tight control of the blocking layers. Preliminary tests on a north Greenland core show improved fitting to the Looyenga density model.

\section{ACKNOWLEDGEMENTS}

We thank R. Laboda for helping with the mechanical design of the instrument and for constructing the electrodes. His thoughtful and precise craftsmanship made the instrument possible.

This is AWI publication 1250.

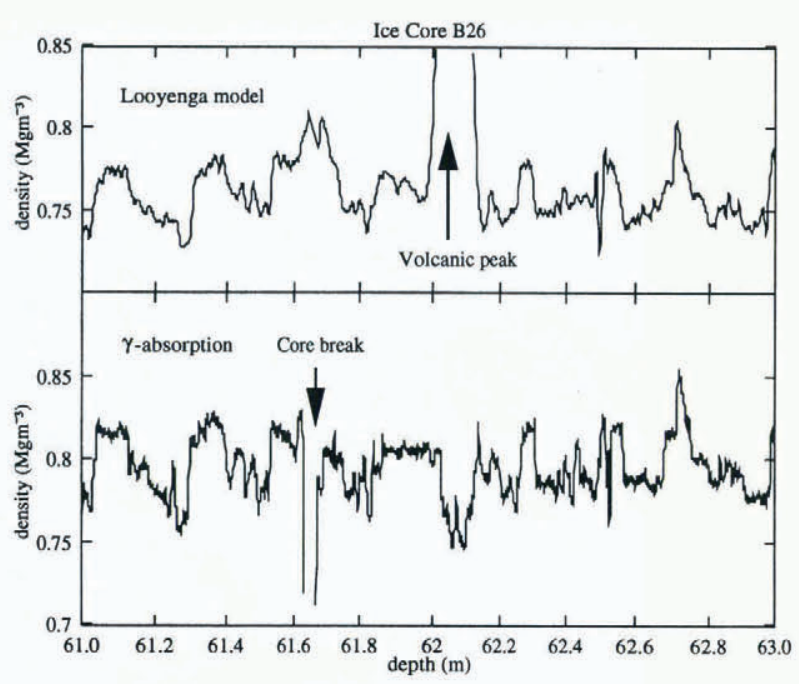

Fig. 8. Density predicted from permittivity by the Looyenga mixing model, compared to density determined from $\gamma$ absorption measurements. The difference at $62.1 \mathrm{~m}$ is due to a volcanichorizon.

\section{REFERENGES}

Camp, P. R., W. Kiszenick and D. Arnold. 1969. Electrical conduction in ice. In Riehl, N., B. Bullemer and H. Engelhardt, eds. Physics of ice. New York, Plenum Publishing Corp., 450-470.

Friedmann, A., J. C. Moore, T. Thorsteinsson, J. Kipfstuhl and H. Fischer. 1995. A 1200 year record of accumulation from northern Greenland. Ann. Glaciol., 21, 1925.

Gross, G.W., C. Wu, L. Bryant and C. McKee. 1975. Concentration dependent solute redistribution at the ice/water phase boundary. 2. Experimental investigation. 7. Chem. Phys., 62 (8), 3085-3092.

Gross, G.W., I. C. Hayslip and R. N. Hoy. 1980. Dielectric relaxation spectrum of ice measured with linear blocking electrodes. Geophysics, 45 (5), 914-927.

Hammer, C. 1977. Past volcanism revealed by Greenland ice sheet impurities. Nature, 270 (5637), 482-486.

Hammer, C. U. 1980. Acidity of polar ice cores in relation to absolute dating, past volcanism, and radio-echoes. f. Glaciol., 25(93), 359-372.

Hammer, C. U., H. B. Clausen and W. Dansgaard. 1980. Greenland ice sheet evidence of post-glacial volcanism and its climatic impact. Nature, 288(5788), 230-235.

Jaccard, C. 1959. Étude théorique et expérimentale des propriétés électriques de la glace. Helv. Phys. Acta, 32 (2), 89-128.

Looyenga, M. 1965. Dielectric constants of heterogeneous mixture. Physica, 31 (3), 401-406.

Moore, J. C. 1993. High-resolution dielectric profiling of ice cores. J. Glaciol., $39(132), 245-248$.

Moore, J. C. and N. Maeno. 1991. Application of the dielectric profiling technique to ice core studies. Proceedings of the NIPR Symposium on Polar Meteorology and Glaciology 4, 81-92.

Moore, J. C. and J. G. Paren. 1987. A new technique for dielectric logging of Antarctic ice cores. 7. Phys. (Paris), 48, Colloq. Cl, 155-160. (Supplément au 3.)

Moore, J., J. Paren and H. Oerter. 1992. Sea salt dependent electrical conduction in polar ice. J. Geophys. Res., 97 (B13), 19,803-19,812.

Mounier, S. and P. Sixou. 1969. A contribution to the study of conductivity and dipolar relaxation in doped ice crystals. In Riehl, N., B. Bullemer and H. Engelhardt, eds. Physics of ice. New York, Plenum Publishing Corp., 562-570.

Paren, J. G. 1973. The electrical behavior of polar glaciers. In Whalley, E., S. J. Jones and L. Gold, eds. Physics and Chemistry of Ice: papers presented at the Symposium on the Physics and Chemistry of Ice, held in Otlawa, Canada, 14-18 August 1972. Ottawa, Ont., Royal Society of Canada, 262267.

Petrenko, V. F. 1993. Electrical properties of ice. CRREL Spec. Rep. 93-20.

Reynolds, J. M. 1985. Dielectric behaviour of firn and ice from the Antarctic Peninsula, Antarctica. f. Glaciol., 31 (109), 253-262.

Wilhelms, F. 1996. Leitfähigkeits- und Dichtemessung an Eisbohrkernen. Ber. Polarforsch. 191

Wolff, E.W., J. C. Moore, H. B. Clausen, C. U. Hammer, J. Kipfstuhl and K. Fuhrer. 1995. Long-term changes in the acid and salt concentrations of the Greenland Ice Core Project ice core from electrical stratigraphy. 7. Geophys. Res., 100 (D8), 16,249-16,263. 